\title{
Lung biopsy in children: when is it useful?
}

Corresponding Author:

Mr Hany Gabra, +441912829867, hany.gabra@newcastle.ac.uk

The Great North Children's Hospital, Department of Paediatric Surgery, Queen

Victoria Road, Newcastle Upon Tyne, NE1 4LP, UK

Authors:

1. Corey David Chan, Newcastle University Medical School, Newcastle upon Tyne, Tyne and Wear, UK

2. Anindya Nyogi, Great North Children's Hospital, Department of Paediatric Surgery, Newcastle upon Tyne, UK

3. Bruce Jaffray, The Newcastle Hospitals NHS Foundation Trust, Department of Paediatric Surgery, The Royal Victoria Infirmary, Newcastle upon Tyne, UK

4. Malcolm Brodlie, Translational and Clinical Research Institute, Faculty of Medical Sciences, Newcastle University and Paediatric Respiratory Medicine, Great North Children's Hospital, Newcastle upon Tyne Hospitals NHS Foundation Trust, UK.

5. Hany Gabra, Great North Children's Hospital, Department of Paediatric Surgery, Newcastle upon Tyne, UK

Keywords: paediatrics; surgery; lung biopsy; lung diseases; diagnostic yield; histology.

Word Count [1198] 


\section{ABSTRACT}

\section{Aim}

To provide a further insight into the usefulness of lung biopsy in children.

\section{Methods}

Lung biopsies in children from January 2007 to December 2017 were reviewed $(n=39)$. The histology results were categorised as: definitive diagnosis, normal lung parenchyma, inconclusive.

\section{Results}

Lung biopsy provided a definitive diagnosis in 25 (64\%) cases. A suspected diagnosis was confirmed in 16 (41\%) and a new diagnosis was found in $9(23 \%)$ children. Histology was inconclusive in 11 (28\%) cases and normal in $3(8 \%)$. Fifteen $(38 \%)$ of children had treatment altered due to the biopsy result.

\section{Conclusion}

Lung biopsy mostly confirmed the suspected diagnosis and was associated with a low procedure related morbidity $(n=1)$ and mortality $(n=0)$. Importantly, the biopsy result identified a pathology which altered treatment in over one third of patients. However, in a number of cases the histology was inconclusive, therefore careful patient selection is recommended to maximise diagnostic yield. 


\section{INTRODUCTION}

Paediatric surgeons are commonly asked to carry out lung biopsies in children to obtain tissue for a definitive histological or microbiological diagnosis. A lung biopsy is an invasive diagnostic procedure however, often performed in children with significant comorbidities.[1] The decision of whether to perform a lung biopsy in this cohort of patients remains an important one, given the risks associated with the surgery. The reported diagnostic yield from lung biopsy is variable, and its utility in bringing a therapeutic change to patients remains largly uncertain.[1, 2] The European protocol for Interstitial Lung Disease in Children suggests performing a lung biopsy only if treatment will be changed as a result of the procedure.[3] Therefore, the selection of appropriate patients is important to maximise utility and avoid unnessessary surgical intervention in vulnerable children. This study aims to determine the diagnostic yield of paediatric lung biopsy in our centre and provide new data surrounding the usefulness of lung biopsy in children.

\section{METHODS}

All children who underwent diagnostic open or thoracoscopic lung biopsy in our centre between January 2007 to December 2017 were identified from clinical coding. All therapeutic lung resections and transbronchial lung biopsies were excluded for the purposes of this study.

In our centre, biopsy samples underwent histological processing by a number of different pathologists and a macroscopic and microscopic report was formulated. Each specimen was routinely processed followed by relevant staining including Gram, Grocott, dPAS and modified Ziehl-Neelseen to identify microorganisms within the sample. When clinical details suggested malignancy, samples underwent immunohistochemical staining for specific markers. In cases of significant uncertainty or rarity, specimens were sent externally for a secondary opinion.

Patient records, histology reports and operation notes were retrospectively reviewed for patient demographics, the indications for biopsy, preoperative investigations, 
surgical technique, complications and the histological outcome. The results from lung biopsy were categorised into three distinct groups: definitive histological diagnosis, normal lung parenchyma, or inconclusive histology. Biopsy results were correlated against the preoperative provisional diagnosis. Medical records were reviewed to determine whether treatment was changed as a result of the histology results.

\section{RESULTS}

Thirty-nine patients were identified for inclusion in the study. Twenty-one were male (53.8\%) and 18 were female (46.2\%). Median age at biopsy was 3.5 years ( 7 days 15 years). There were 33 open procedures and six thoracoscopic (15\%). Median operative time for open biopsy was 61 mins (31-81), and for thoracoscopic biopsy was 83 mins (30-125). The median duration of a chest drain following surgery was 2 days (1-4). The complications associated with the procedure included one child who had a haemothorax. No persistent air leak or pneumothorax was reported in this cohort.

Four out of the 39 lung biopsy samples were sent for a second histological review at other specialist UK centres. In all four of these cases, the second opinion agreed with the original decision but provided further histological detail and supplementary information.

Lung biopsy provided a definitive histological diagnosis in 25 (64\%) children, of which a suspected diagnosis was confirmed in $16(41 \%)$ children, and a new diagnosis was found in $9(23 \%)$. In the remaining children, 11 (28\%) cases had a histology result which was inconclusive and $3(8 \%)$ had normal histology (Table 1). 
Table 1: Histological results from lung biopsy

Single suspected preoperative diagnosis

CONFIRMATIVE OF SUSPECTED PREOPERATIVE DIAGNOSIS

Preoperative diagnosis

PTLD

Pulmonary Hypertension

Lymphangiectasis

Metastasis

Lung dysplasia

IPEX $X^{\circ}$ associated pneumonia

Histology

NEW HISTOLOGICAL DIAGNOSIS

Preoperative diagnosis

Fungal infection

Alveolar capillary dysplasia

Metastatic disease

Pseudomonas infection

NORMAL LUNG PARENCHYMA

Preoperative diagnosis

Parenchymal lung disease

CPAM $^{\circ}$

INCONCLUSIVE HISTOLOGY

Preoperative diagnosis

Infection NOS

Fungal infection

$E B V^{d}$ lymphoproliferation

PTLD

Pulmonary Hypertension

Lymphangiectasis

Wilms Tumour metastasis

Alveolar capillary dysplasia

Features of immunodeficiency

\begin{tabular}{|l|}
\hline $\mathbf{N}$ \\
\hline 3 \\
\hline 2 \\
\hline 2 \\
\hline 1 \\
\hline 1 \\
\hline 1 \\
\hline \multirow{3}{*}{$N=10$} \\
\end{tabular}

Histology

Chronic granulomatous disease

Chronic diffuse pneumonitis

Acute haemorrhagic infarction

Miliary tuberculosis

Multiple preoperative differential diagnoses

CONFIRMATIVE OF A SUSPECTED PREOPERATIVE DIAGNOSIS

Preoperative diagnoses

PTLD/infection NOS

Fungal infection/Residual Lymphoma

Chemotherapy reactive lesions/Fungal

infection

Fungal infection/mycobacterial infection

Lymphoma/Fungal

infection/Mycobacterial

Lymphoma/infection NOS

NEW HISTOLOGICAL DIAGNOSIS

Preoperative diagnoses

Pulmonary Hypertension/Surfactant

protein B deficiency

NORMAL LUNG PARENCHYMA

Preoperative diagnoses

Lymphoproliferation/Bronchiolitis

obliterans

Histology

Normal

Normal

\begin{tabular}{|l|l|}
\hline $\mathbf{N}$ \\
\hline 2 \\
\hline 1 \\
\hline 1 \\
\hline 1
\end{tabular} \mid \begin{tabular}{l}
\multirow{3}{*}{$N=5$} \\
\end{tabular}

Histology

Non-specific changes

Non-specific interstitial pneumonitis

Non-specific changes

INCONCLUSIVE HISTOLOGY

Preoperative diagnoses

Fungal infection/PTLD

GvHD/Fungal infection/CMV $/ T B^{g}$

Histology

PTLD

Diffuse Large B-Cell Lymphoma

Chemotherapy-related interstitial

pneumonitis

Aspergillus infection

Non-tuberculous mycobacterial

infection

Classical Hodgkin's Lymphoma

Histology

Pulmonary interstitial glycogenesis

No specific preoperative diagnosis

NEW HISTOLOGICAL DIAGNOSIS

Indication for biopsy

Interstitial infiltrate on $C X R^{h}$

Respiratory condition of new-born of

unknown aetiology

Persistent pulmonary infiltrates on CXR

INCONCLUSIVE HISTOLOGY

Indication for biopsy

Respiratory distress of unknown

aetiology

Unknown cause of chronic lung disease

Query cause of pneumonia

\begin{tabular}{l|l} 
Abnormal CT chest opacification & Non-specific changes
\end{tabular}

${ }^{a}$ Post-transplant lymphoproliferative disorder, ${ }^{b}$ immunodysregulation polyendocrinopathy enteropathy $X$-linked syndrome, ${ }^{C}$ Congenital
malformation, ${ }^{d}$ Epstein-Barr virus, ${ }^{\circ}$ graft versus host disease, ${ }^{c}$ cytomegalovirus, ${ }^{g}$ tuberculosis, ${ }^{n}$ Chest $X$-ray, ${ }^{\prime}$ computerised tomography

\begin{tabular}{|c|c|c|}
\hline Histology & $\mathbf{N}$ & \multirow{2}{*}{$N=1$} \\
\hline Normal & 1 & \\
\hline Histology & $\mathbf{N}$ & \multirow{3}{*}{$N=2$} \\
\hline Non-specific thrombi related disease & 1 & \\
\hline Non-specific pneumonitis & 1 & \\
\hline
\end{tabular}

Non-specific thrombi related

Histology

Interstitial pneumonitis

Pulmonary interstitial glycogenesis

Necrotising pneumonia

Histology

Mild non-specific changes

Minimal interstitial non-specific fibrosis

No specific evidence of cause 
In the cases where the histology was inconclusive, $64 \%$ had a preoperative indication querying an infective cause. In seven children, no specific preoperative diagnosis was stated, and in this group, $57 \%$ of histology reports were inconclusive. When the indication for biopsy was a suspected neoplasm, a definitive diagnosis was achieved in $90 \%$ of cases, making it the indication with the highest diagnostic yield.

Fifteen (38\%) children had their treatment altered due to the biopsy results. In these cases, the biopsy result identified a new diagnosis or confirmed one of the several suspected diagnoses that were queried pre-operatively, and therefore guided management.

Thirteen (33\%) of the patients died in a median of 85 days (1-666 days) post biopsy. These deaths were not directly attributable to the surgical procedure, and rather reflect the severe nature and complexity of such conditons which warrant a lung biopsy. A large number of our patients had complex and rare conditions which made them extremely sick and vulnerable. Two (5\%) of our patients died within 30 days of surgery. The first child was a 6-week old baby who died the day after the biopsy due to legionella pneumonia with lung necrosis. The second child was a 2-week old baby with alveolar capillary dysplasia, who died on the second postoperative day.

\section{DISCUSSION}

In our centre, lung biopsy is performed thoracoscopically for older children where intercostal space allows $10 \mathrm{~mm}$ trocar for the staplers. For smaller children, the thoracotomy approach is used. CT-guided percutaneous lung biopsy has been successful in children,[4] however, there is a significant radiation risk which must be considered. Therefore, open or thoracoscopic lung biopsy remain the standard procedure in our centre.

Although all specimens obtained from biopsy were suitable for histological analysis, a definite diagnosis was reached in only $64 \%$ of cases. This is lower than some studies, which report close to an $80 \%$ diagnosis rate.[5] This may be because a 
significant proportion of our patients are immunocompromised with diffuse lung lesions, greatly complicating their pathologies and treatments. Although morbidity and mortality from paediatric lung biopsies are reported to be high, $[2,5]$ in our study only one haemothorax was noted as a procedure-related morbidity with zero procedure related mortality, supporting the relative safety of lung biopsy as a diagnostic investigation.

In four cases the biopsy sample was sent externally for a second opinion. Paediatric lung disorders form a heterogeneous group, with each individual possessing complex and rare pathologies. In our study, although all second opinions agreed with the original pathologists' conclusion, they provided supplementary knowledge and further histological detail. A nationwide approach to paediatric lung histology may provide a higher diagnostic yield through the process of learning across specialist centres, given the rarity of such conditions.

In our series, lung biopsy provided a definitive histological diagnosis in $64 \%$ of cases. In $41 \%$ of cases, a preoperative suspected diagnosis was confirmed. Therefore, if the preoperative workup is strongly suggestive of a diagnosis, an additional lung biopsy may not be necessary. However, in $23 \%$ of children, lung biopsy provided a new diagnosis which was not suspected preoperatively. Of this group, $6(67 \%)$ had a suspected pre-operative diagnosis that was proven incorrect on biopsy, and 3 (33\%) had no definitive preoperative diagnosis. In $28 \%$ of cases, the results from lung biopsy were inconclusive which did not aid management, and further clinical and radiological investigations were recommended. Therefore, surgeons and paediatricians must carefully select patients for lung biopsy, especially in groups which were shown to have a lower diagnostic yield. 


\section{CONCLUSION}

In our cohort, lung biopsy mostly confirmed a suspected preoperative diagnosis and was associated with a low procedure related morbidity $(n=1)$ and mortality $(n=0)$. Importantly, the biopsy result identified a pathology which altered treatment in over one third of patients. However, in a number of cases the histology was inconclusive. A higher incidence of inconclusive histology was identified when the indication for biopsy queried an infective cause or when no specific preoperative diagnosis was stated. Therefore, careful patient selection is recommended to maximise diagnostic yield.

\section{Funding Statement:}

This research received no specific grant from any funding agency in the public, commercial or not-for-profit sectors. Malcolm Brodlie was supported by a Medical Research Council Clinician Scientist Fellowship (MR/M008797/1).

\section{Competing Interests:}

Non-declared.

\section{Contributorship Statement:}

1. Corey David Chan was the lead author of this paper and undertook the vast majority of the data collection, data processing and data analysis.

2. Anindya Nyogi assisted with data collection and data processing and contributed significantly to the writing of the paper.

3. Bruce Jaffray offered guidance and significant input into the writing of the discussion of this paper.

4. Malcolm Brodlie contributed to the writing of this paper and provided a significant input of clinical knowledge.

5. Hany Gabra was the overall supervisor of the project and assisted in facilitating the data collection and contributed to the writing of the paper.

What is already known on this topic: 
Lung biopsy in children is used as a diagnostic tool and is associated with significant morbidity and mortality.

Lung biopsy is performed in children who have very serious and complicated conditions.

The diagnostic yield of lung biopsy in children is variable and limited guidelines exist on when to carry out this procedure.

\section{What this study adds:}

Lung biopsy results altered treatment in $38 \%$ of children.

Paediatric lung biopsy was associated with a low procedure related morbidity $(n=1)$ and mortality $(n=0)$.

A higher rate of inconclusive histology was identified when looking for an infective cause or when no specific preoperative diagnosis was stated.

\section{REFERENCES}


1. Lamoshi AY, Nakayama DK. Usefulness of lung biopsy in pediatric pulmonary conditions. The American surgeon. 2015;81:31-3 Online First: 2015/01/09].

2. Naiditch JA, Barsness KA, Rothstein DH. The Utility of Surgical Lung Biopsy in Immunocompromised Children. The Journal of Pediatrics. 2013;162:133-6.e1 doi: https://doi.org/10.1016/i.jpeds.2012.06.019

3. Bush A, Cunningham S, De Blic J, et al. European protocols for the diagnosis and initial treatment of interstitial lung disease in children. Thorax. 2015:thoraxjnl-2015-207349 Online.

4. Naumann H, Pittaway A, Lynn AM, Vo NJ. CT-guided percutaneous lung biopsy under general anesthesia: a pediatric case series and literature review. Paediatric anaesthesia. 2012;22:469-75 doi: 10.1111/j.1460-9592.2011.03773.x [published Online First: 2012/01/04].

5. Greenhalgh RM, Yardley IE, Child F, Bruce J, Humphrey GM. Lung biopsy for chronic pulmonary disease in children. Journal of Pediatric Surgery. 2014;49:1075-7 doi: https://doi.org/10.1016/j.jpedsurg.2013.10.026 